\title{
Wearable Intelligent Systems for E-Health
}

\author{
Carmen C. Y. Poon and Qing Liu
}

Joint Research Centre for Biomedical Engineering, Department of Electronic Engineering, The Chinese University of Hong Kong, Hong Kong SAR

cpoon@ee.cuhk.edu.hk, qingliu@ee.cuhk.edu.hk

\author{
Hui Gao and Wan-Hua Lin \\ SIAT-Institute of Biomedical and Health Engineering, Chinese Academy of Sciences, Shenzhen, China \\ hui.gao@siat.ac.cn,wh.lin@siat.ac.cn
}

\section{Yuan-Ting Zhang*}

Joint Research Centre for Biomedical Engineering, Department of Electronic Engineering, The Chinese University of Hong Kong, Hong Kong SAR; SIAT-Institute of Biomedical and Health Engineering \& Key Laboratory for Health Informatics, Chinese Academy of Sciences, Shenzhen, China ytzhang@ee.cuhk.edu.hk

\begin{abstract}
Due to the increasingly aging population, there is a rising demand for assistive living technologies for the elderly to ensure their health and well-being. The elderly are mostly chronic patients who require frequent check-ups of multiple vital signs, some of which (e.g., blood pressure and blood glucose) vary greatly according to the daily activities that the elderly are involved in. Therefore, the development of novel wearable intelligent systems to effectively monitor the vital signs continuously over a 24 hour period is in some cases crucial for understanding the progression of chronic symptoms in the elderly. In this paper, recent development of Wearable Intelligent Systems for e-Health (WISEs) is reviewed, including breakthrough technologies and technical challenges that remain to be solved. A novel application of wearable technologies for transient cardiovascular monitoring during water drinking is also reported. In particular, our latest results found that heart rate increased by 9 bpm $(\mathrm{P}<0.001)$ and pulse transit time was reduced by $5 \mathrm{~ms}(\mathrm{P}<$ 0.001 ), indicating a possible rise in blood pressure, during swallowing. In addition to monitoring physiological conditions during daily activities, it is anticipated that WISEs will have a number of other potentially viable applications, including the real-time risk prediction of sudden cardiovascular events and deaths.
\end{abstract}

Category: Smart and intelligent computing

Keywords: Wearable intelligent system; E-Health; Cardiovascular monitoring; Water drinking

\section{INTRODUCTION}

The global population of elderly people has been growing rapidly over the past few decades. In Europe, one third of the population will be over 65 years old by the year 2035 [1]. China, on the other hand, is one of the countries faced with the fastest growing aging problem. Over one-fifth of the world's elderly population (aged 65 and over) lives in China and this proportion is envisaged to increase up to $24 \%$ by 2020 . Furthermore, the problem is aggravated by the fact that the number of elderly people leading solitary lives is also increasing and that a large percentage of them are suffering from either chronic diseases or varying degrees of disability.

Chronic diseases have been reckoned to be the world's lead-

This is an Open Access article distributed under the terms of the Creative Commons Attribution Non-Commercial License (http://creativecommons.org/licenses/ by-nc/3.0/) which permits unrestricted non-commercial use, distribution, and reproduction in any medium, provided the original work is properly cited.

Received 30 July 2011, Accepted 30 August 2011

*Corresponding Author 
ing cause of death and disability, and will account for almost three-fourths of all deaths by 2020. Each year, the number of deaths caused by cardiovascular diseases and hypertension is estimated to be 16.7 million and 7.1 million respectively. The population of diabetic adults is expected to reach 300 million by 2025 [2]. The cumulative effects of all these factors will be serious social problems, which in turn will place a severe financial burden on healthcare services. As a result, there is an increasing demand for a shift from the conventional hospital-centered healthcare system for treating symptoms to a new healthcare system that emphasizes early detection of risk factors, early diagnosis, and early treatment [3].

In order to collect health information before symptoms of illnesses appear, new acquisition systems are needed. In addition to systems for acquiring high-resolution images and genetic information, Wearable Intelligent Systems for e-Health (WISEs) have been proposed because of their capability for exercising long-term, continuous, and unobtrusive collection of health information. Since some of the physiological parameters (e.g. blood pressure and blood glucose) vary greatly according to the daily activities that the subjects are involved in, the development of novel WISEs that can effectively monitor the vital signs continuously over a long period is in some cases crucial to understanding the progression of some chronic symptoms in the elderly. WISEs can also capture transient features and early indicators of health deterioration.

The objectives of this paper are 1) to review recent development of WISEs, including breakthrough technologies and technical challenges that remain to be solved and 2) to report the results of a novel application of the wearable technologies in capturing transient cardiovascular features during water drinking. The rest of paper is organized as follows: Section III reviews the state-ofthe-art of WISEs. Section IV discusses the development of textile sensors, which can be considered as a major breakthrough technology in WISEs. Section V focuses on the problems that remain to be solved for WISEs, i.e., the issue of motion artifacts and potential techniques for reducing them. Section VI reports the latest results of our recent study on a novel application of wearable technologies, which is to measure the transient variations of multiple cardiovascular parameters (i.e., heart rate and pulse transit time) during water drinking. Finally, the conclusions and other potentially viable applications of wearable medical devices are presented in Section VII.

\section{STATE-OF-THE-ART OF WISES}

WISEs consist of various kinds of sensors such as microelectronic, biochemical, or textile-based sensors embedded or integrated into wearable objects (such as gloves, finger rings, wristband, ear-worn devices, waist-belt, or cloth, etc.) for the noninvasive monitoring of physiological signals and parameters, such as electrocardiogram (ECG), biopotentials, photoplethysmogram (PPG), heart rate (HR), blood pressure (BP), blood glucose (BG), and blood oxygen saturation $\left(\mathrm{SpO}_{2}\right)$, respiration and body activity. Recently, a wearable system incorporating an ultrasound sensor [4] has also been developed for the cardiopulmonary activity monitoring in emergency.

Health information acquired by WISEs can be used for real- time, on-site detection of physiological conditions of the elderly and can also be transferred wirelessly to remote medical centers for early diagnosis. By the same token, remote medical centers can, in turn send early treatment advice to the patients in realtime.

WISEs should entail five characteristics, namely MINDS [5]: 1) Miniaturized: to include innovative approaches and measurement principles for acquiring physiological information as well as to incorporate small-sized and low-power integrated circuit designs; 2) Intelligent: to be able to learn and timely evaluate an individual's health conditions, and issue alerts whenever appropriate; 3) Networked: to connect on-body sensors by a body area network (BAN) and to wirelessly hook up with remote medical centers; 4) Digitalized: to incorporate new bio-sampling and bio-signal processing theories to handle the massive multi-modal health information; and 5) Standardized: to allow standard communication and evaluation protocols for ensuring interoperability, measurement accuracy and information integrity.

A number of WISEs have been developed by various research groups, including a smart garment for measuring HR, breathing rate (BR), body temperature (BT), and $\mathrm{SpO}_{2}[6]$ an earlobe PPG detector [7], a wrist-worn device AMON for $\mathrm{BP}, \mathrm{ECG}, \mathrm{SpO}_{2}$ monitoring [8], a glove for monitoring skin temperature (SKT), PPG, and galvanic skin response (GSR) [9], a respiration waist belt [10] and a ring-type PPG sensor [11].

The main research focus of our team has been on the development of a cuff-less BP estimation technique based on the measurement of pulse transit time (PTT). The technology has been implemented as a cuff-less BP watch previously by one of our industrial sponsors [12] and as an h-shirt developed

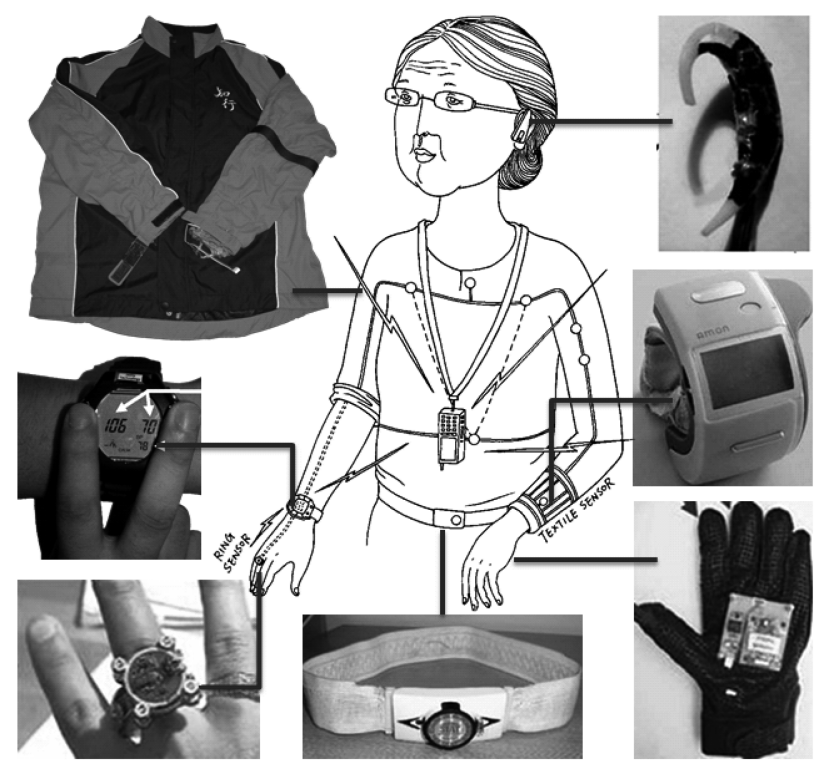

Fig. 1. Device prototypes in wearable intelligent systems for e-Health WISEs) by different research groups. Clockwise from the upper right hand corner are: an earlobe photoplethysmogram (PPG) detector [7], a wristworn device AMON for blood pressure (BP), electrocardiogram (ECG), blood oxygen saturation monitoring [8], a glove for monitoring skin temperature, PPG, and galvanic skin response [9], a respiration waist belt [10], a ring-type PPG sensor [11], a cuff-less BP watch [12] produced by Jetfly Technology Ltd. Based on the technology developed in our research center and an h-shirt developed in our research center for monitoring PPG, ECG, and BP [13]. 
by our research center for monitoring PPG, ECG, and BP [13].

Fig. 1 shows some of the device prototypes that can be used in WISEs. In order to develop these prototypes, new sensors and innovative measurement principles as opposed to the conventional medical devices used in the hospitals are often incorporated, as summarized below.

\section{A. Electrocardiogram}

Non-contact wireless ECG sensors based on the principle of capacitive coupling are now becoming washable and fully integrated with clothing and wearable accessories [14-16]. These wireless sensors overcome the shortcomings of traditional wet adhesive electrodes and can operate without directly contacting the skin surface. The sensors can be manufactured in the form of fabric by weaving or knitting conductive yarn/rubber/ink electrodes [17].

In addition to arrhythmia, HR and heart rate variability (HRV) are also indicators of health [18-20]. HR and HRV data can be extracted from ECG, PPG, remotely by microwave radar sensors based on the doppler effect [21] and most recently by applying independent component analysis (ICA), a blind source separation method, on video images of people's faces [22].

\section{B. Respiration}

Respiration is most commonly measured by sensors integrated into a belt or garment. The types of sensors used include impedance pneumographic, inductive plethysmographic, piezoresistive, piezoelectric and textile-based capacitive sensors [23-25]. Respiration rate can also be extracted from other physiological signals such as ECG and PPG [26].

\section{C. $\mathrm{SpO}_{2}$}

The most popular method for non-invasive estimation of $\mathrm{SpO}_{2}$ is by means of photoplethysmography. The method is based on the difference in absorption of two wavelengths of light by the pulsatile arteriolar blood flow. Sensors have been integrated into finger rings, earlobe devices, foreheads, wristworn devices and shirts in wearable application [11, 16, 17]. A wearable imaging device is also able to detect $\mathrm{SpO}_{2}$ and blood volume non-invasively by functional near-infrared (fNIR) spectroscopy [27].

\section{Blood Glucose}

Diabetes is a common disease in the elderly population. In particular, sufferers of type I diabetes require daily BG measurements followed by insulin injections. These patients' quality of life can be greatly improved by using feedback system with a small insulin pump to regulate the insulin delivery based on the measured BG levels. The system requires the patient's glycemia to be measured accurately and continuously such that the insulin infusion rate and dosage can be adjusted accordingly. In recent years, several approaches for continuous monitoring have been developed, such as the subcutaneous needle sensor. Fig. 2 shows a needle-type glucose sensor used for a wearable artificial endocrine pancreas [28]. This sensor is placed in the

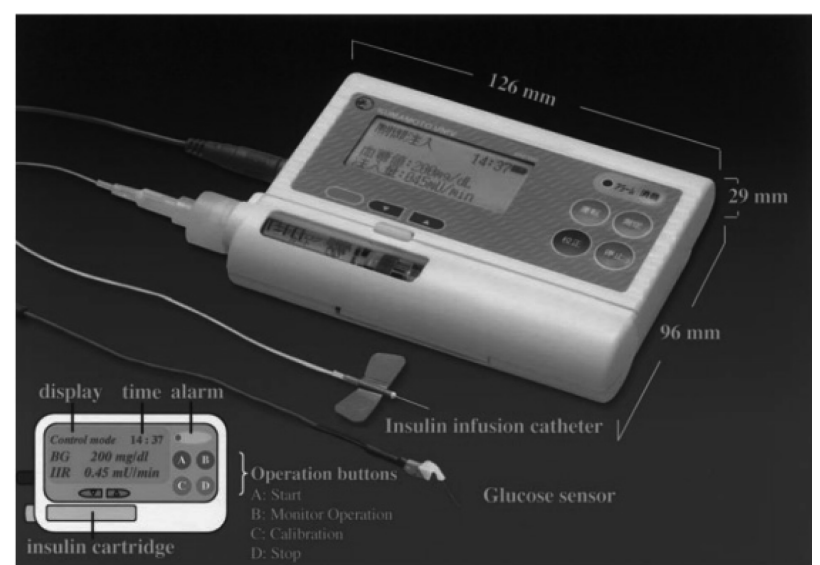

Fig. 2. A wearable artificial endocrine pancreas with a needle-type glucose sensor [28].

subcutaneous tissue and measures subcutaneous glucose concentration continuously. Another study reported a wearable glucose monitor based on SC open-flow microperfusion techniques, including handling of liquids, glucose sensors and electronics for motor control, sensor read-out, and communication [29]. Nevertheless, the above methods are invasive.

Advanced technologies for BG monitoring focus on needlefree, transcutaneous measurements [30]. A number of methods have been demonstrated to have great potential for the noninvasive and continuous monitoring of BG, e.g., by reverse iontophoresis, impedance spectroscopy, photoacoustic spectroscopy, near infrared spectroscopy, electrophoresis, enzyme-based direct electron transfer [30,31], some of which have been implemented in "watch-like" wrist-worn devices.

\section{E. Other Biochemical Measurements}

In addition to BG, biochemical measurements of other body fluids, such as blood, sweat and urine, are also under active development. Real-time monitoring of the $\mathrm{pH}$ of sweat is usually performed using wearable micro-fluidic devices [32, 33]. The microchip was fabricated using polymer and can also be manufactured in textile form [32].

\section{F. Blood Pressure}

Hypertension is another common disease found in the elderly population. Elevated BP increases the workload of the heart and scars the artery walls. Increases in either BP or BP variability (BPV) are partly responsible for various cardiovascular events [34]. Nevertheless, most individuals with hypertension experience no symptoms, which often make them overlook their ailment. Thus, early detection of BP for health condition assessment by wearable devices before a severe event occurs is very important.

Technologies advanced in wearable BP monitoring focuses on continuous and noninvasive measurement without using a cuff. Cuff-less BP can be measured from the radial pulse waveform by arterial tonometry [35]. Another promising technique for cuff-less BP is based on the estimation of PTT $[12,36]$. Such technologies can be integrated with a personal 


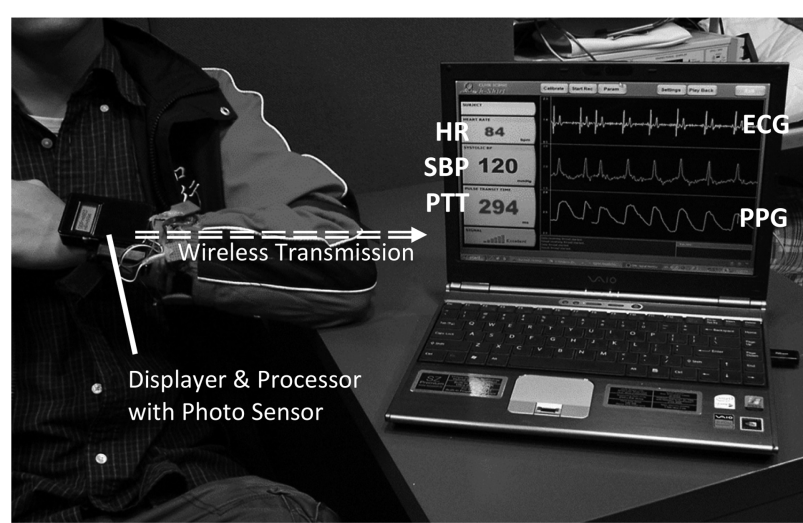

Fig. 3. An h-shirt-based wearable intelligent systems for e-Health (WISEs) prototype developed in our research center for monitoring electrocardiogram, photoplethysmogram, and cuff-less blood pressure based on the estimation of pulse transit time [13].

digital assistant (PDA), a watch and a finger ring for cuffless monitoring of arterial BP [12, 35, 36]. Together with electronic textile (e-textile)-based technologies, the technique can also be used to design a shirt for long-term, hands-free continuous monitoring of BP, as shown in Fig. 3 [13].

\section{E-TEXTILE SENSORS FOR WISES}

A breakthrough technique adopted in many WISEs is the electronic textile (e-textile) sensing technology, which has led to a number of innovative methods for measuring various physiological signals and parameters, as reviewed in Section II. Conductive and functional materials in the form of fiber and yarn can now be integrated into garments and used as sensors and connectors [14]. Smart shirts based on utilization of etextiles having the same appearance as common clothes are capable of vital signs monitoring without causing deterioration of fabric behavior [37]. Table 1 summarizes typical usage of etextile sensors in health monitoring.

\section{A. Conductive Materials}

Conductive yarn, conductive rubber, and conductive ink have been developed into sensors [23] or used as an interconnection substrate [37] in WISEs. Conductive yarns and fibers are made by mixing pure metallic or natural fibers with conductive materials [24]. Pure metallic yarns can be made of composite stainless steel or fine continuous conductive metal-alloy [17]. Combination of fibers with conductive materials can be completed by the following methods [37]: 1) Fibers filled with conductive material (e.g., carbon or metallic particles); 2) Fibers coated with conductive polymers or metal; and 3) Fibers spun with thin metallic or plastic conductive threads. Currently, most textile-based sensors (fabric sensors) for health monitoring are achieved by knitting or embroidering conductive yarns [23]. Conductive rubber is made by filling a silicon rubber with conductive materials such as carbon or metals. They are usually called carbon or metal loaded rubber (CLR/MLR) [23, 38], and can be integrated into textile substrates by coating technologies. Researchers at North Carolina State University proposed another method of making textile electrodes for WISEs, which is by silk-screen printing conductive inks ( $\mathrm{Ag}$ or $\mathrm{AgCl})$ onto non-woven substrates [17].

The conductive properties are exploited in order to capture physiological electric activity (such as ECG, electroencephalogram and electromyogram) from the surface of the skin [23, 39-41]. Impedance pneumography for monitoring respiration is accomplished by locating two e-textile sensors on the chest, and inputting a low-current high-frequency sinusoidal signal between the sensors. Impedance across the chest was evaluated by measuring the voltage drop during breathing [23, 42]. This is the long-established standard method for respiration monitoring

Table 1. E-textile sensors in health monitoring

\begin{tabular}{|c|c|c|c|}
\hline Devices implementation & Sensing conponents & Signals & Applications \\
\hline Woven or knitted conductive yarn/ rubber/ ink electrodes & Fabric sensors & Electrocardiogram & Cardiopulmonary \\
\hline Woven or knitted conductive yarn/ rubber electrodes & Fabric sensors & Electromyography & Neural rehabilitation \\
\hline Woven or knitted conductive yarn/ rubber electrodes & $\begin{array}{l}\text { Impedance pneumographic } \\
\text { sensors }\end{array}$ & Respiration & Cardiopulmonary \\
\hline Textile fibers or small-sized strips based on conductive yarn & $\begin{array}{l}\text { Inductive plethysmograhic } \\
\text { sensors }\end{array}$ & Respiration & Cardiopulmonary \\
\hline $\begin{array}{l}\text { Textile fibers or small-sized strips based on } \\
\text { conductive yarn/ carbon filled rubber/ electro active polymer }\end{array}$ & Piezoresistive sensors & Respiration & Cardiopulmonary \\
\hline EAP based textile fibers or small-sized strips & Piezoresistive sensors & Movement and posture & Neural rehabilitation \\
\hline EAP based textile fibers or small-sized strips & Piezoelectric sensors & $\begin{array}{l}\text { Carotid pulse, } \\
\text { radial artery pulse, } \\
\text { heart apex pulse, and sound }\end{array}$ & Cardiopulmonary \\
\hline Optical fibers & Optical fibers & Pulse oxygen & Cardiopulmonary \\
\hline EAP based textile fibers or small-sized strips & Thermoelectric sensors & Skin tempreture & Neural rehabilitation \\
\hline Woven or knitted conductive yarn/ rubber/ \& optical fibers & Fabric sensors & Cuffless blood pressure & Cardiopulmonary \\
\hline
\end{tabular}


[23]. Another new method for respiration monitoring is based on inductive plethysmography. The respiration pattern is assessed by measuring the change in inductance with an inductive sensor, which is usually designed in the form of a belt, constituting a wire formed in a sinusoidal pattern $[23,43]$. Continuous cuffless BP monitoring based on bio-models has been studied based on measurement of PTT from ECG obtained by textile electrodes and PPG by a photoreflective sensor [13].

\section{B. Functional Materials}

Piezoresistive, piezoelectric, chemoresistive, thermoelectric, photoelectric materials and other functional materials in the form of textile fibers can be used for sensors, actuators electronic components, and power sources based on its physical effect [24]. The changes of the resistance of piezoresistive sensors when stretched and strained were used to evaluate biomechanical variables (such as respiration, movement, gesture and posture) $[23,44]$. Carbon filled with rubber was successfully used for making a piezoresistive respiration sensor [23]. It is usually designed in the form of elastic strip.

The piezoelectric effect refers to the generation of current when stress is applied. Several kinds of accelerometers based on piezoelectric, piezoresistive materials have been widely used for physical activity monitoring, movement and fall detection $[44,45]$. Piezoelectric sensors made of electroactive polymer (EAP) based textile fiber, can be used for monitoring carotid pulse, radial artery pulse, heart apex pulse, and sound detection [24]. These can also be applied as wearable power sources, textile keyboards, and general-purpose contact sensors (e.g., fall detectors) [24].

\section{Integration of Textile-based Sensors with WISEs}

Integration of textile-based sensors into clothing for health monitoring dates back to 1996 [46], when the Georgia Tech Wearable Motherboards was one of the first health applications integrated in a garment. The motherboard created a system for a soldier that is capable of providing unobtrusive monitoring of biological signals through textile sensors.

Another example of wearable health monitoring is the LifeShirt, which is a multi-function ambulatory system monitoring key physiological measurements, such as ECG, ventilation, respiratory rate, heart rate and activity, during daily living [29]. Other famous projects in this field include the MIThril LiveNet System [47], which is aimed at developing new techniques of human-computer interaction for body-worn applications. Since 2002, there have been a cluster of European Commission cofinanced projects on Smart Fabrics, Interactive Textiles (SFITs), as shown in Fig. 4, starting with "physiological parameteroriented projects" (e.g., WEALTHY, MyHeart, MERMOTH, and OFSETH) relying on textile-embedded sensors, and extending to biosensing textiles (BIOTEXs) [48], and a combination of signals (e.g., PROETEX) [49]. Currently, only a few wearable e-textile health monitoring systems (e.g., the VivoMetrics LifeShirt, Sensatex SmartShirt system, and MagIC) have been commercialized [15], while several more are still under development [23]

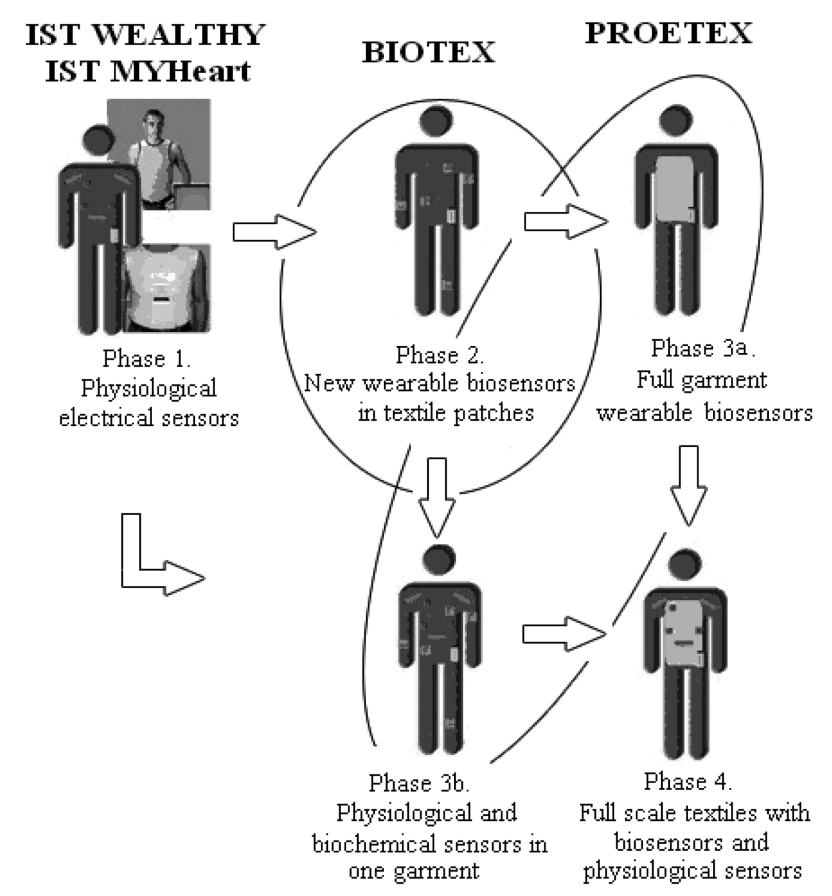

Fig. 4. Cluster of European Commission co-financed projects on smart fabrics, interactive textiles, reproduced from [49]. IST: Information Society Technologies.

\section{MOTION ARTIFACT REDUCTION}

A technical challenge unique to WISEs is the accurate measurement during user movement. The signals acquired by wearable sensors are often corrupted by a patient's motion. This motion artifact (MA) results in inaccurate estimation of required parameters, even when patient movements are very small, such as shivering. Generally, artifacts arising from skin stretch and the electrodes or sensors are the major causes of motion artifact [44]. At present, various methods have been studied for removing artifacts.

The most well-known method of motion artifact reduction is adaptive filtering [50-53]. Additional motion tracking sensor and signal processing procedures are needed for reducing motion artifacts. The signal acquired by this motion sensor becomes the reference input for the adaptive filter. The MIT group utilized a MEMS accelerometer as a reference channel to reconstruct the signals obtained from their finger-ring PPG sensor [50]. Another study compares the use of the force-sensitiveresistor sensor and the accelerometer as a reference of motion and found that the former better characterizes the probe-coupling artifacts between the skin and a wrist-type device [52].

The adaptive algorithms commonly used include the least mean square (LMS) and the recursive least squares adaptive methods. A study [45] compares the performance of these two algorithms and found that both algorithms produced similar improvements. Nevertheless, implementing the adaptive LMS algorithm is advantageous, since it requires significantly fewer operations. The advantages of adaptive filter methods include faster response time and better real-time performance, but additional sensors are required to detect the noise reference signal. More recently, a new method was reported that uses a synthetic 
noise reference signal generated from an MA corrupted PPG signal without any extra hardware such as accelerometers or additional source-detector pairs for noise reference signal generation [54].

Another common method is to use the signal processing algorithms directly, such as by wavelet transform and ICA. In past decades, the wavelet transform has been proven to be a useful tool for signal analysis [55]. The wavelet transform can be used as a decomposition of a signal in the time-frequency and time-scale planes. The general denoising procedure involves three steps: decomposition, thresholding, and reconstruction [56]. A comparative analysis [57] used different wavelets for the case of MA reduction from corrupted PPG signals, including Daubechies, biorthogonal, reverse biorthogonal, symlet, Coiflet types of wavelets. Experimental results revealed that the Daubechies wavelet resulted in excellent reduction of MA compared to other wavelets. However, the study in [58] revealed that wavelet transformation has limited applicability in restoring corrupted PPG signals for both HR and PTT measurements.

Recently, increased emphasis has been placed on ICA to reduce motion artifacts, because it does not require prior knowledge of the system $[59,60]$. This method assumes that all source signal component pairs are mutually independent. A recent work, however, indicates that motion significantly affects arterial flow and therefore, care must be taken when applying ICA to light-based sensor data acquired from wearable platforms [61].

Some other techniques have also been found to be effective for reducing motion artifacts, including the smoothed pseudo Wigner-Ville distribution [62], the cycle-by-cycle Fourier series [63], the empirical mode decomposition [64] and the singular value decomposition method [65].

The minimum correlation discrete saturation transform (MCDST) algorithm based on an optical model derived from photon diffusion analysis was considered to be more robust under low Signal-to-Noise Ratios than the clinically verified motion-resistant algorithm discrete saturation transform (DST). Further, the experiment with varying motion severity demonstrates that MCDST has a slightly better performance than the DST algorithm [66]. Another experiment compared the LMS adaptive filter and the exponentially weighted least square (EWLS) adaptive filter with the MCDST. The experimental results indicate that both adaptive filters can perform better than the MCDST, and the EWLS adaptive filter performs better than the LMS adaptive filter in motion noise reduction [67].

Although there have been many attempts, the reduction of motion artifacts is still a major limitation in most practical implementations of WISEs. Further studies of the generation mechanisms of physiological signals and motion artifacts as well as their interactions are essential to development of a model-based approach for reducing motion artifacts in order to acquire real-time health information by WISEs.

\section{APPLICATION: CAPTURING TRANSIENT CARDIOVASCULAR FEATURES DURING WATER DRINKING}

One of the biggest advantages of using WISEs is that they can supply transient features during daily activities such as swallowing. Swallowing disorders frequently occur in the elderly and monitoring during the swallowing process is therefore of interest for assistive living technologies for the elderly. In this study, preliminary results for continuous monitoring of HR and PTT, which is commonly acknowledged to be inversely related to $\mathrm{BP}$, during water drinking are presented.

\section{A. Protocol}

Seven healthy subjects (including 4 males and 3 females) participated in the experiment. The subjects were $26 \pm 6$ years old, $171 \pm 8 \mathrm{~cm}$ tall and weighed $66 \pm 7 \mathrm{~kg}$. All subjects were non-smokers. The subjects were required to refrain from eating or drinking 2.5 hours before the experiment.

The experiment was conducted in the supine position. ECG and PPG data were collected from the subjects' wrists and left index finger respectively using an in-house system. A microphone sensor was placed on the subjects' throat to capture sound induced during the moment of swallowing. The signals were all sampled at $1 \mathrm{kHz}$.

Upon arrival, the subjects were asked to rest in the supine position for 10 minutes. They were then asked to drink water through a straw, by taking a single swallow once every minute on 5 occasions.

\section{B. Signal Processing}

All data trials were performed on a beat-to-beat basis. The R$\mathrm{R}$ interval (RRI) was determined as the time lapse between the successive R peaks of ECG. PTT was measured as the time interval between the peaks of ECG R waves and the first derivative of PPG signals in the same cardiac circle. The HR was calculated from the RRI in beats per minute (bpm).

Swallows were characterized by finding peaks in the acoustic signals captured by the microphone. There were 5 datasets for each subject, each containing data for a single swallow. Data for a 30-second interval before and after each swallow was segmented. In total, 35 datasets, each for a 1 minute interval, were obtained. All the datasets were then interpolated to $1 \mathrm{~Hz}$ and averaged. The difference between parameters taken during swallowing (i.e., the peak value) and during the baseline interval (i.e., during the first second) was statistically analyzed by using the paired Student's t-test. A P-value less than 0.001 is considered as statistically significant.

\section{Results and Discussion}

Fig. 5 plots the averaged response of HR and PTT induced by a single swallow for the 35 datasets. Significant and transient increases in HR and decreases in PTT were observed during swallowing during 30 seconds $(\mathrm{P}<0.001)$. The standard deviation of HR and PTT during each second varies from 6.9 $\mathrm{bpm}$ to $10.5 \mathrm{bpm}$, and from $15.1 \mathrm{~ms}$ to $18.5 \mathrm{~ms}$, respectively, before swallowing; and it subsequently varies from $8.4 \mathrm{bpm}$ to $12.1 \mathrm{bpm}$ and $14.6 \mathrm{~ms}$ to $18.1 \mathrm{~ms}$.

Swallowing is a complex and highly coordinated activity involving muscles of the tongue, larynx, pharynx and esophagus [68]. Previous study has found chronotropic increase of HR 

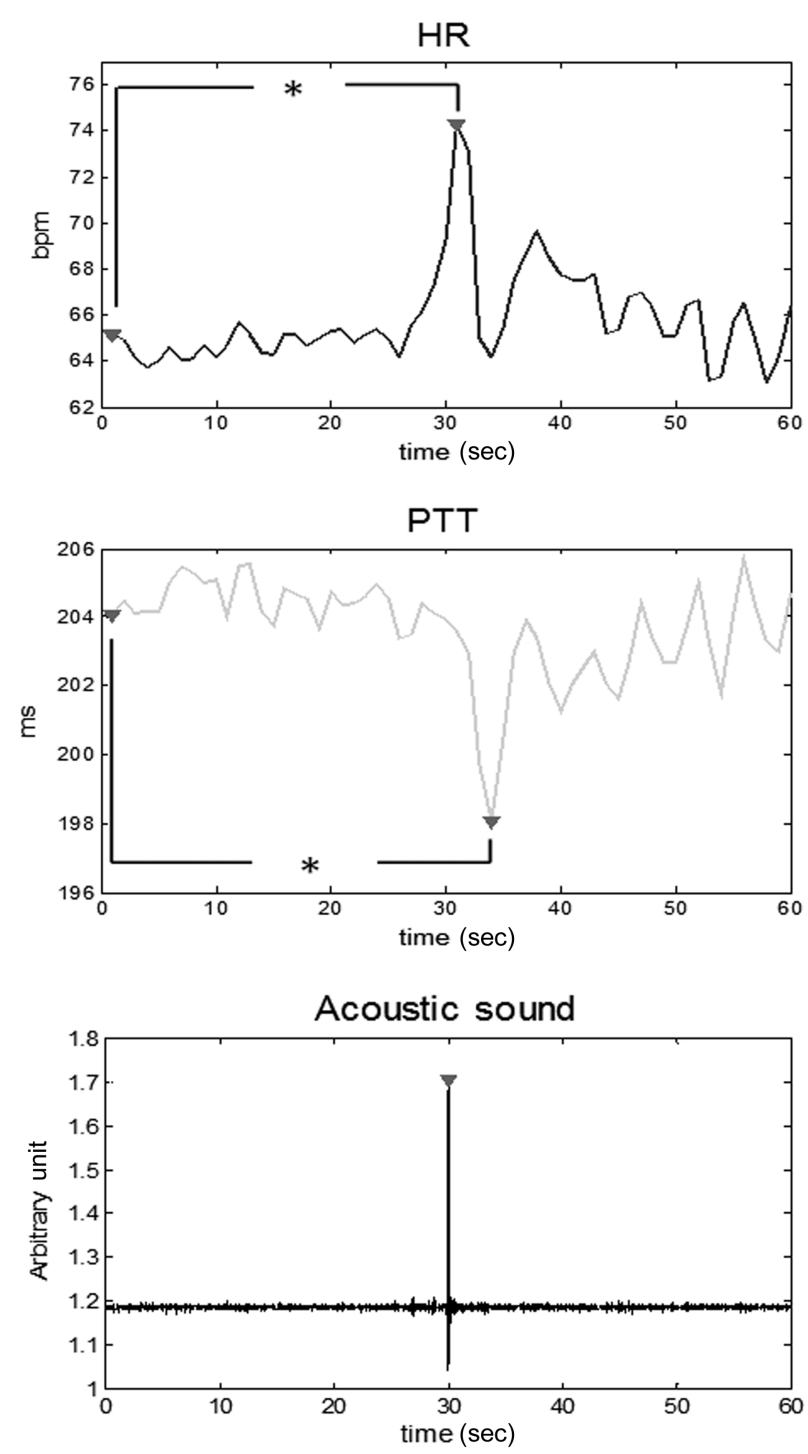

Fig. 5. Averaged response of heart rate $(H R)$, pulse transit time (PTT) and acoustic sound for a single swallow in 35 datasets ( $\left.{ }^{*} P<0.001\right)$.

for a single swallow and a series of three or more swallows [68]. There was also a rapid and significant increase in BP during water drinking [69]. These simultaneous cardiovascular responses were generated by the feedback from the pharyngeal and esophageal receptors. A feedforward mechanism by a central descending signal from the higher brain centers may also contribute to these responses.

PTT is inversely related to BP by the Moens-Korteweg equation. In this study, a transient but pronounced decrease was observed in PTT in addition to an increase in HR, indicating that a transient increase in BP is induced by a single swallow. These abrupt responses could be attributed to inhibition of vagal activity during swallowing, since sympathetic signals cannot be transmitted over such a short interval (1-2 secs) due to the slow adrenergic synapses [68]. The influence of sympathetic activity on PTT needs to be further investigated by introducing a series of swallows rather than a single swallow.

\section{CONCLUSIONS}

Driven by an increasingly aging population, the prevalence of chronic diseases, and continuously rising healthcare costs, a new health paradigm that emphasizes early detection, early diagnosis and early treatment, is highly recommended. In this new paradigm, WISEs have been identified as crucial components to provide noninvasive and continuous monitoring to assist the lives of the elderly and other chronic patients. In the past few decades, the development of WISEs has experienced several major technological advancements, including emerging technology for e-textile sensors. Nevertheless, there are a number of technical challenges that remain to be solved for WISEs. This includes a complete solution to motion artifact reduction in real-life, real-time measurements.

The applications of wearable technologies are wide ranging. In this study, the latest measurement results of transient surges in HR and BP, as indicated by the reduction in PTT, during swallowing have been presented. The results indicate the possibility of monitoring swallowing in the elderly.

Another potentially viable application of WISEs will be to predict near-term risk of cardiovascular diseases, which is another chronic disease commonly found in the elderly. A considerable number of cardiovascular disease patients die suddenly without prior symptoms and therefore, wearable technologies can play a major role in identifying transient activity-related features to potentially predict near-term risks of cardiovascular events or deaths.

\section{ACKNOWLEDGEMENTS}

This work was supported in part by the Hong Kong Innovation Technology Fund (ITF), the 973 Project Fund (2010CB732606) and the Guangdong LCHT Innovation Research Team Fund in China. The authors are grateful to Standard Telecommunication Ltd., Bird International Ltd. Bright Steps Corporation and PCCW for their support of the ITF projects.

The authors are grateful to Mr. Laurence Chan and Mr. Billy Leung for their contributions to the development of the prototype of the h-shirt, and Ms. Yali Zheng for her contribution to the data collection of the presented monitoring experiment during water drinking.

\section{REFERENCES}

1. N. Noury, G. Virone, P. Barralon, J. Ye, V. Rialle, and J. Demongeot, "New trends in health smart homes," Proceedings of the 5th International Workshop on Enterprise Networking and Computing in Healthcare Industry, Santa Monica, CA, 2003, pp. 118127.

2. American Heart Association, "International Cardiovascular Disease Statistics," http://www.americanheart.org/.

3. X. F. Teng, Y. T. Zhang, C. C. Y. Poon, and P. Bonato, "Wearable medical systems for p-health," IEEE Reviews in Biomedical Engineering, vol. 1, pp. 62-74, 2008.

4. A. Lanata, E. Pasquale Scilingo, and D. De Rossi, “A multimodal transducer for cardiopulmonary activity monitoring in emer- 
gency," IEEE Transactions on Information Technology in Biomedicine, vol. 14, no. 3, pp. 817-825, May 2010.

5. Y. T. Zhang, Y. S. Yan, and C. C. Y. Poon, "Some perspectives on affordable healthcare systems in China," Proceedings of the 29th Annual International Conference of IEEE Engineering in Medicine and Biology Society, Lyon, France, 2007, p. 6154.

6. D. Curone, E. L. Secco, A. Tognetti, G. Loriga, G. Dudnik, M. Risatti, R. Whyte, A. Bonfiglio, and G. Magenes, "Smart garments for emergency operators: the ProeTEX project," IEEE Transactions on Information Technology in Biomedicine, vol. 14, no. 3, pp. 694-701, May 2010.

7. L. Wang, B. P. Lo, and G. Z. Yang, "Multichannel reflective PPG earpiece sensor with passive motion cancellation," IEEE Transactions on Biomedical Circuits and Systems, vol. 1, no. 4, pp. 235-241, 2007.

8. U. Anliker, J. A. Ward, P. Lukowicz, G. Troster, F. Dolveck, M. Baer, F. Keita, E. B. Schenker, F. Catarsi, L. Coluccini, A. Belardinelli, D. Shklarski, M. Alon, E. Hirt, R. Schmid, and M. Vuskovic, "AMON: a wearable multiparameter medical monitoring and alert system," IEEE Transactions on Information Technology in Biomedicine, vol. 8, no. 4, pp. 415-427, Dec. 2004.

9. D. W. Ryoo, Y. S. Kim, and J. W. Lee, "Wearable systems for service based on physiological signals," Proceedings of the 27th Annual International Conference of the Engineering in Medicine and Biology Society, Shanghai, China, 2005, pp. 2437-2440.

10. G. Z. Liu, B. Y. Huang, and L. Wang, "A wearable respiratory biofeedback system based on generalized body sensor network," Telemedicine and e-Health, vol. 17, no. 5, pp. 348-357, 2011.

11. H. H. Asada, P. Shaltis, A. Reisner, S. Rhee, and R. C. Hutchinson, "Mobile monitoring with wearable photoplethysmographic biosensors," IEEE Engineering in Medicine and Biology Magazine, vol. 22, no. 3, pp. 28-40, 2003.

12. C. C. Y. Poon, Y. M. Wong, and Y. T. Zhang, "M-health: the development of cuff-less and wearable blood pressure meters for use in body sensor networks," IEEE/NLM Life Science Systems and Applications Workshop, Bethesda, MD, 2006.

13. Y. T. Zhang, C. C. Y. Poon, C. H. Chan, M. W. W. Tsang, and K. F. Wu, "A health-shirt using e-textile materials for the continuous and cuffless monitoring of arterial blood pressure," Proceedings of the 3rd IEEE-EMBS International Summer School and Symposium on Medical Devices and Biosensors, Cambridge, MA, 2006, pp. 86-89.

14. R. Paradiso, G. Loriga, N. Taccini, A. Gemignani, and B. Ghelarducci, "WEALTHY--a wearable healthcare system: new frontier on e-textile," Journal of Telecommunications and Information Technology, vol. 4, pp. 105-113, 2005.

15. K. Heimann, M. Steffen, N. Bernstein, N. Heerich, S. Stanzel, A. Cordes, S. Leonhardt, T. G. Wenzl, and T. Orlikowsky, "Noncontact monitoring of heart and lung activity using magnetic induction measurement in a neonatal animal model," Biomedizinische Technik, vol. 54, no. 6, pp. 337-345, 2009.

16. Y. M. Chi, T. P. Jung, and G. Cauwenberghs, "Dry-contact and noncontact biopotential electrodes: Methodological review," IEEE Reviews in Biomedical Engineering, vol. 3, pp. 106-119, 2010.

17. T. H. Kang, "Textile-embedded sensors for wearable physiological monitoring systems," Ph.D. dissertation, North Carolina State University, Raleigh, NC, 2006.

18. P. Denes, J. C. Larson, D. M. Lloyd-Jones, R. J. Prineas, and P. Greenland, "Major and minor ECG abnormalities in asymptom- atic women and risk of cardiovascular events and mortality," Journal of the American Medical Association, vol. 297, no. 9, pp. 978-985, Mar. 2007.

19. M. T. Cooney, E. Vartiainen, T. Laakitainen, A. Juolevi, A. Dudina, and I. M. Graham, "Elevated resting heart rate is an independent risk factor for cardiovascular disease in healthy men and women," American Heart Journal, vol. 159, no. 4, pp. $612-$ 619.e3, 2010.

20. T. D. Miller, "The exercise treadmill test: Estimating cardiovascular prognosis," Cleveland Clinic Journal of Medicine, vol. 75, no. 6, pp. 424-430, 2008.

21. S. Suzuki, T. Matsui, H. Imuta, M. Uenoyama, H. Yura, M. Ishihara, and M. Kawakami, "A novel autonomic activation measurement method for stress monitoring: non-contact measurement of heart rate variability using a compact microwave radar," Medical and Biological Engineering and Computing, vol. 46, no. 7, pp. 709-714, 2008.

22. M. Z. Poh, D. J. McDuff, and R. W. Picard, "Non-contact, automated cardiac pulse measurements using video imaging and blind source separation," Optics Express, vol. 18, no. 10, pp. 1076210774, 2010

23. C. R. Merritt, "Electronic textile-based sensors and systems for long-term health monitoring," Ph.D. dissertation, North Carolina State University, Raleigh, NC, 2008.

24. F. Carpi and D. De Rossi, "Electroactive polymer-based devices for e-textiles in biomedicine," IEEE Transactions on Information Technology in Biomedicine, vol. 9, no. 3, pp. 295-318, 2005.

25. C. R. Merritt, H. T. Nagle, and E. Grant, "Textile-based capacitive sensors for respiration monitoring," IEEE Sensors Journal, vol. 9, no. 1, pp. 71-78, 2009.

26. S. Dash, K. H. Shelley, D. G. Silverman, and K. H. Chon, "Estimation of respiratory rate from ECG, photoplethysmogram, and piezoelectric pulse transducer signals: a comparative study of timefrequency methods," IEEE Transactions on Biomedical Engineering, vol. 57, no. 5, pp. 1099-1107, 2010.

27. M. Izzetoglu, K. Izzetoglu, S. Bunce, H. Ayaz, A. Devaraj, B. Onaral, and K. Pourrezaei, "Functional near-infrared neuroimaging," IEEE Transactions on Neural Systems and Rehabilitation Engineering, vol. 13, no. 2, pp. 153-159, 2005.

28. S. Ichimori, K. Nishida, S. Shimoda, T. Sekigami, Y. Matsuo, K. Ichinose, M. Shichiri, M. Sakakida, and E. Araki, "Development of a highly responsive needle-type glucose sensor using polyimide for a wearable artificial endocrine pancreas," Journal of Artificial Organs, vol. 9, no. 2, pp. 105-113, 2006.

29. R. Dudde, T. Vering, G. Piechotta, and R. Hintsche, "Computeraided continuous drug infusion: setup and test of a mobile closedloop system for the continuous automated infusion of insulin," IEEE Transactions on Information Technology in Biomedicine, vol. 10, no. 2, pp. 395-402, 2006.

30. C. M. Girardin, C. Huot, M. Gonthier, and E. Delvin, "Continuous glucose monitoring: a review of biochemical perspectives and clinical use in type 1 diabetes," Clinical Biochemistry, vol. 42, no. 3, pp. 136-142, 2009.

31. C. E. Ferrante do Amaral and B. Wolf, "Current development in non-invasive glucose monitoring," Medical Engineering and Physics, vol. 30, no. 5, pp. 541-549, 2008.

32. F. Benito-Lopez, S. Coyle, R. Byrne, A. Smeaton, N. E. O'Connor, and D. Diamond, "Pump less wearable microfluidic device for real time $\mathrm{pH}$ sweat monitoring (in Proceedings of the Euro- 
sensors XXIII conference)," Procedia Chemistry, vol. 1, no. 1, pp. 1103-1106, Sep. 2009.

33. F. Benito-Lopez, S. Coyle, R. Byrne, and D. Diamond, "Sensing sweat in real-time using wearable microfluidics," Proceedings of the 7th International Workshop on Wearable and Implantable Body Sensor Networks, Singapore, 2010.

34. P. M. Rothwell, S. C. Howard, E. Dolan, E. O'Brien, J. E. Dobson, B. Dahlof, P. S. Sever, and N. R. Poulter, "Prognostic significance of visit-to-visit variability, maximum systolic blood pressure, and episodic hypertension," Lancet, vol. 375, no. 9718, pp. 895905, Mar. 2010

35. K. G. Ng, C. M. Ting, J. H. Yeo, K. W. Sim, W. L. Peh, N. H. Chua, N. K. Chua, and F. Kwong, "Progress on the development of the MediWatch ambulatory blood pressure monitor and related devices," Blood Pressure Monitoring, vol. 9, no. 3, pp. 149-165, 2004.

36. P. Shaltis, A. Reisner, and H. Asada, "Calibration of the photoplethysmogram to arterial blood pressure: capabilities and limitations for continuous pressure monitoring," Proceedings of the 27th Annual International Conference of the Engineering in Medicine and Biology Society, Shanghai, China, 2005, pp. 39703973.

37. G. Troster, T. Kirstein, and P. Lukowicz, "Wearable computing: packaging in textiles and clothes," Proceedings of the 14th European Microelectronics and Packaging Conference and Exhibition, Friedrichshafen, Germany, 2003.

38. R. Wijesiriwardana, K. Mitcham, and T. Dias, "Fibre-meshed transducers based real time wearable physiological information monitoring system," Proceedings Eighth International Symposium on Wearable Computers, Arlington, VA, 2004, pp. 40-47.

39. E. P. Scilingo, A. Gemignani, R. Paradiso, N. Taccini, B. Ghelarducci, and D. De Rossi, "Performance evaluation of sensing fabrics for monitoring physiological and biomechanical variables," IEEE Transactions on Information Technology in Biomedicine, vol. 9, no. 3, pp. 345-352, 2005.

40. C. Linti, H. Horter, P. Osterreicher, and H. Planck, "Sensory baby vest for the monitoring of infants," Proceedings of the International Workshop on Wearable and Implantable Body Sensor Networks, Cambridge, MA, 2006, pp. 135-137.

41. C. Y. Ryu, S. H. Nam, and S. Kim, "Conductive rubber electrode for wearable health monitoring," Proceedings of the 27th Annual International Conference of the Engineering in Medicine and Biology Society, Shanghai, China, 2005, pp. 3479-3481.

42. R. Paradiso, G. Loriga, and N. Taccini, "A wearable health care system based on knitted integrated sensors," IEEE Transactions on Information Technology in Biomedicine, vol. 9, no. 3, pp. 337-344, 2005

43. N. Noury, A. Dittmar, C. Corroy, R. Baghai, J. L. Weber, D. Blanc, F. Klefstat, A. Blinovska, S. Vaysse, and B. Comet, "VTAMN: a smart clothe for ambulatory remote monitoring of physiological parameters and activity," Proceedings of the 26th Annual International Conference of the IEEE Engineering in Medicine and Biology Society, San Francisco, CA, 2004, pp. 3266-3269.

44. J. G. Webster, "Reducing motion artifacts and interference in biopotential recording," IEEE Transactions on Biomedical Engineering, vol. BME-31, no. 12, pp. 823-826, 1984.

45. G. Comtois, Y. Mendelson, and P. Ramuka, "A comparative evaluation of adaptive noise cancellation algorithms for minimizing motion artifacts in a forehead-mounted wearable pulse oxime- ter," Proceedings of the 29th Annual International Conference of IEEE Engineering in Medicine and Biology Society, Lyon, France, 2007, pp. 1528-1531.

46. "Georgia Tech Wearable Motherboard"M: The Intelligent Garment for the 21st Century," http://www.gtwm.gatech.edu/.

47. B. R. Chen, G. Peterson, G. Mainland, and M. Welsh, "LiveNet: using passive monitoring to reconstruct sensor network dynamics," Distributed Computing in Sensor Systems: 4th IEEE International Conference, DCOSS 2008 Santorini Island, Greece, June 11-14, 2008 Proceedings. Lecture Notes in Computer Science Vol. 5067, S. Nikoletseas, B. Chlebus, D. Johnson, and B. Krishnamachari, Eds., Heidelberg: Springer Berlin, pp. 79-98, 2008.

48. S. Coyle, K. T. Lau, N. Moyna, D. O'Gorman, D. Diamond, F. Di Francesco, D. Costanzo, P. Salvo, M. G. Trivella, D. E. De Rossi, N. Taccini, R. Paradiso, J. A. Porchet, A. Ridolfi, J. Luprano, C. Chuzel, T. Lanier, F. Revol-Cavalier, S. Schoumacker, V. Mourier, I. Chartier, R. Convert, H. De-Moncuit, and C. Bini, "BIOTEX--biosensing textiles for personalised healthcare management," IEEE Transactions on Information Technology in Biomedicine, vol. 14, no. 2, pp. 364-370, Mar. 2010.

49. Centre Suisse d'Electronique et Microtechnique SA, "SFIT Cluster of EC Co-financed projects," http://csnej106.csem.ch/Sfit/ $\mathrm{Html} /$ Projects.Html.

50. H. H. Asada, H. H. Jiang, and P. Gibbs, "Active noise cancellation using MEMS accelerometers for motion-tolerant wearable bio-sensors," Proceedings of the 26th Annual International Conference of the IEEE Engineering in Medicine and Biology Society, San Francisco, CA, 2004, pp. 2157-2160.

51. P. F. Wei, R. W. Guo, J. M. Zhang, and Y. T. Zhang, "A new wristband wearable sensor using adaptive reduction filter to reduce motion artifact," Proceedings of the 5th International Conference on Information Technology and Applications in Biomedicine in conjunction with 2nd International Symposium and Summer School on Biomedical and Health Engineering, Shenzhen, China, 2008, pp. 278-281.

52. C. K. Park, J. C. Sohn, J. H. Kim, and H. J. Choi, "Artifactresistant design of a wrist-type heart rate monitoring device," Proceedings of the 11th International Conference on Advanced Communication Technology, Pyeongchang, Korea, 2009, pp. $2313-$ 2316.

53. W. C. Lee, Y. S. O. Yang, T. C. Ke, C. S. Wei, and H. C. Lee, "Adaptive reduction of motion artifact in a portable ECG system," Proceedings of the 9th IEEE Sensors Conference, Waikoloa, HI, 2010, pp. 704-707.

54. M. R. Ram, K. V. Madhav, E. H. Krishna, K. N. Reddy, and K. A. Reddy, "On the performance of Time Varying Step-size Least Mean Squares(TVS-LMS) adaptive filter for MA reduction from PPG signals," Proceedings of the International Conference on Communications and Signal Processing, Kerala, India, 2011, pp. 431-435.

55. C. M. Lee and Y. T. Zhang, "Reduction of motion artifacts from photoplethysmographic recordings using a wavelet denoising approach," Proceedings of the IEEE EMBS Asian-Pacific Conference on Biomedical Engineering, Kyoto, Japan, 2003, pp. 194195.

56. M. Alfaouri and K. Daqrouq, "ECG signal denoising by wavelet transform thresholding," American Journal of Applied Sciences, vol. 5, no. 3, pp. 276-281, 2008. 
57. M. Raghuram, K. Venu Madhav, E. Hari Krishna, and K. Ashoka Reddy, "On the performance of wavelets in reducing motion artifacts from photoplethysmographic signals," Proceedings of the 4th International Conference on Bioinformatics and Biomedical Engineering, Chengdu, China, 2010.

58. J. Y. A. Foo, "Comparison of wavelet transformation and adaptive filtering in restoring artefact-induced time-related measurement," Biomedical Signal Processing and Control, vol. 1, no. 1, pp. 93-98, 2006.

59. B. S. Kim and S. K. Yoo, "Motion artifact reduction in photoplethysmography using independent component analysis," IEEE Transactions on Biomedical Engineering, vol. 53, no. 3, pp. 566568, 2006.

60. J. Y. A. Foo, "Use of independent component analysis to reduce motion artifact in pulse transit time measurement," IEEE Signal Processing Letters, vol. 15, pp. 124-126, 2008.

61. J. Yao and S. Warren, "A short study to assess the potential of independent component analysis for motion artifact separation in wearable pulse oximeter signals," Proceedings of the 27th Annual International Conference of the Engineering in Medicine and Biology Society, Shanghai, China, 2005, pp. 3585-3588.

62. Y. S. Yan, C. C. Y. Poon, and Y. T. Zhang, "Reduction of motion artifact in pulse oximetry by smoothed pseudo Wigner-Ville distribution," Journal of NeuroEngineering and Rehabilitation, vol. 2, pp. 3, Mar. 2005.

63. K. A. Reddy, B. George, and V. J. Kumar, "Use of Fourier series analysis for motion artifact reduction and data compression of photoplethysmographic signals," IEEE Transactions on Instru- mentation and Measurement, vol. 58, no. 5, pp. 1706-1711, 2009.

64. Q. Wang, P. Yang, and Y. Zhang, "Artifact reduction based on Empirical Mode Decomposition (EMD) in photoplethysmography for pulse rate detection," Proceedings of the 32nd Annual International Conference of the IEEE Engineering in Medicine and Biology Society, Buenos Aires, Argentina, 2010, pp. 959962.

65. K. A. Reddy and V. J. Kumar, "Motion artifact reduction in photoplethysmographic signals using singular value decomposition," IEEE Instrumentation and Measurement Technology Conference on Synergy of Science and Technology in Instrumentation and Measurement, Warsaw, Poland, 2007.

66. Y. S. Yan and Y. T. Zhang, "An efficient motion-resistant method for wearable pulse oximeter," IEEE Transactions on Information Technology in Biomedicine, vol. 12, no. 3, pp. 399-405, 2008.

67. S. Kunchon, T. Desudchit, and C. Chinrungrueng, "Comparative evaluation of adaptive filters in motion artifact cancellation for pulse oximetry," Proceedings of the 5th International Colloquium on Signal Processing and Its Applications, Kuala Lumpur, Malaysia, 2009, pp. 307-311.

68. O. P. Sherozia, V. V. Ermishkin, and E. V. Lukoshkova, "Dynamics of swallowing-induced cardiac chronotropic responses in healthy subjects," Bulletin of Experimental Biology and Medicine, vol. 135, no. 4, pp. 322-326, 2003.

69. Y. Endo, K. Yamauchi, Y. Tsutsui, Z. Ishihara, F. Yamazaki, S. Sagawa, and K. Shiraki, "Changes in blood pressure and muscle sympathetic nerve activity during water drinking in humans," Japanese Journal of Physiology, vol. 52, no. 5, pp. 421-427, 2002.
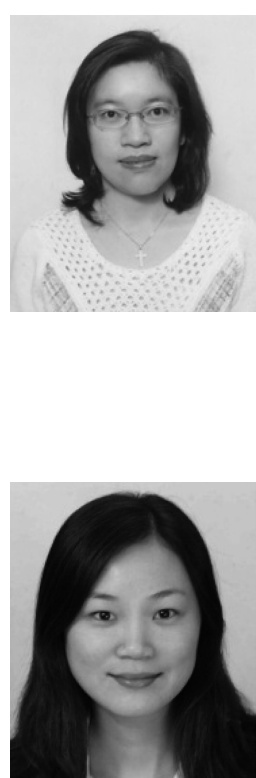

\section{Carmen C. Y. Poon}

Carmen C. Y. Poon received her B.A.Sc. in Engineering Science (Biomedical Option) and her M.A.Sc. at the University of Toronto, Canada. She completed her Ph.D. at The Chinese University of Hong Kong, where she now works as a Research Assistant Professor. She serves as a theme chair of the 33rd Annual International Conference of the IEEE Engineering in Medicine and Biology Society (EMBC) and the Managing Editor of the IEEE Trans. on Information Technology in BioMedicine (T-ITB). She was a Guest Editor for the T-ITB Special Issue on Body Sensor Networks and is now co-editing another T-ITB Special Issue on Atherosclerotic Cardiovascular Health Informatics. Her current research interests include wearable medical devices, body area/sensor networks (BAN/BSN), BSN security, biometrics, bio-modelling, physiological signal processing, $\mathrm{p}$-Health and mobile Health technologies, and cardiovascular health informatics.

\section{Hui Gao}

Hui Gao received the B.E. and M.E. degrees from Tianjin Medical University, Tianjin, China, in 2007 and 2010, respectively. She is currently a Research Assistant of the Key Lab of Health Informatics of Chinese Academy of Sciences, Shenzhen, China, and SIAT-Institute of Biomedical and Health Engineering, Chinese Academy of Sciences, Shenzhen, China. Her current research interests include multi-mode physiological signal processing and cardiovascular health informatics. 

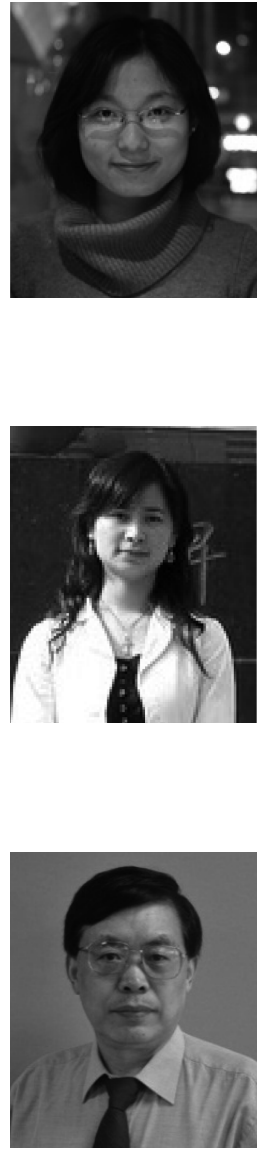

\section{Yuan-Ting Zhang}

Yuan-Ting Zhang is currently the Head of the Division of Biomedical Engineering at the Chinese University of Hong Kong. He also serves as the Director of the Key Laboratory for Health Informatics of the Chinese Academy of Sciences, and the Director of the SIAT-Institute of Biomedical and Health Engineering of Chinese Academy of Science. He chaired the Biomedical Division of Hong Kong Institution of Engineers in 1996/97 and 2000/01. Dr. Zhang was the Technical Program Chair of the 20th IEEE-EMBS Annual Int'I Conference in 1998 and the General Conference Chair of the 27th IEEE-EMBS Annual Int'I Conference in 2005. He was elected as an AdCom member in 1999 and served as Vice-President (Conferences) of IEEE-EMBS in 2000. Dr. Zhang serves currently as a member of IEEE-EMBS Technical Committee of Wearable Systems and Sensors, and the Editor-in-Chief for IEEE TRANSACTIONS ON INFORMATION TECHNOLOGY IN BIOMEDICINE. Dr. Zhang's research interests include neural engineering, wearable medical devices, and body sensor networks particularly for mobile health. He has published more than 300 scientific articles in the area of biomedical engineering and filed over 30 patent applications. Dr. Zhang and his team received numerous awards and recognitions which include the Fellow of International Academy of Medical and Biological Engineering, IEEE Fellow, AIMBE Fellow, the APICT2009 Award, and the recipient of 2006 Distinguished Service Award of IEEE-EMBS. 